\title{
Existence-uniqueness of solutions for fuzzy nabla initial value problems on time scales
}

\author{
R. Leelavathi' ${ }^{1}$ G. Suresh Kumar' ${ }^{1}$, M.S.N. Murty ${ }^{2}$ and R.V.N. Srinivasa Rao ${ }^{3 *}$ (1)
}

${ }^{\text {"Correspondence: }}$

rvnrepalle@gmail.com

${ }^{3}$ Department of Mathematics, College of Natural and

Computational Sciences, Wollega University, Nekemte, Ethiopia

Full list of author information is

available at the end of the article

\begin{abstract}
This paper is devoted to studying the existence and uniqueness of solutions of fuzzy nabla dynamic equations on time scales under Hukuhara differentiability. We apply the Banach contraction mapping principle to establish existence and uniqueness solution for fuzzy nabla initial value problems on time scales and also obtain existence result using Ascoli's theorem and Schauder's fixed point theorem.
\end{abstract}

MSC: 03E72; 26E70; 34A07

Keywords: Time scales; Hukuhara difference; Nabla Hukuhara derivative; Fuzzy differential equations

\section{Introduction}

In modeling a real world phenomenon, some ambiguity or inaccuracy happens because of inadequate data about the parameters which cannot actually portray the idea of the problem. In order to manage this inaccuracy or ambiguity Zadeh [32], presented the theory called fuzzy sets. The fuzzy set theory is an excellent approach which helps us to deal with fuzzy dynamic models. Fuzzy set theory is the best source to study fuzzy differential equations (FDEs) or interval differential equations. FDEs play a vital role in applications of biology, economics, and many other engineering problems where uncertainty arises. Hukuhara [15] initiated the difference between two sets called Hukuhara difference and developed the theory of derivatives and integrals for set-valued mappings. Later, Puri and Ralescu [26] studied Hukuhara derivative for fuzzy functions using Hukuhara difference, and it is the primary approach for studying uncertainty of the dynamical systems. Further, Kaleva [17] studied FDEs under Hukuhara differentiability and also studied the existence and uniqueness of the solutions to FDEs using Hukuhara derivative which has a drawback that the solutions exist only when the functions have an increasing length of support. To overcome this circumstance, Bede and Gal [7] studied generalizations of the differentiability of fuzzy number-valued functions. Later, Stefanini and Bede [27] studied generalized Hukuhara differentiability of interval-valued functions and interval differential equations. Further, Malinowski $[24,25]$ studied the concept of second type Hukuhara derivative for interval differential equations and interval Cauchy problem with second type Hukuhara derivative. Furthermore, Zhang and Sun [36] studied the stability of FDEs under second type Hukuhara derivative.

(c) The Author(s) 2019. This article is distributed under the terms of the Creative Commons Attribution 4.0 International License (http://creativecommons.org/licenses/by/4.0/), which permits unrestricted use, distribution, and reproduction in any medium, provided you give appropriate credit to the original author(s) and the source, provide a link to the Creative Commons license, and indicate if changes were made. 
The theory of time scales was initiated by the German mathematician Stefan Hilger [13]. The important features of time scales are extension, unification, and generalization. The fundamental theory and applications on time scales calculus and dynamic equations on time scales are found in $[1,10]$. The theory of time scale calculus is applicable to any field in which a dynamic process incorporates both discrete or continuous time models. Alternative solutions of linear dynamic equations on time scales and boundary value problems for dynamic equations on time scales were studied in [4] and [11]. Oscillatory behavior of solutions to different classes of dynamic equations has been a subject of numerous studies; see, for instance, the papers [2, 8, 9, 20,33, 35]. Existence of solutions and convergence results for dynamic initial value problems using lower and upper solutions were studied by [34].

Some recent studies and applications in economics [5], production, inventory models [6], adaptive control [16], neural networks [21], and cellular neural networks [12] suggested that nabla derivative is more preferable than delta derivative on time scales.

Hukuhara differentiability of interval-valued functions and interval differential equations on time scales was studied in [23]. Hong et al. [14] studied differentiability for multivalued functions on time scales. Vasavi et al. [28-31] introduced and studied fuzzy dynamic equations on time scales. Recently, Leela et al. $[18,19]$ developed nabla Hukuhara differentiability and integrability on time scales. With the importance and advantages of nabla derivative, we propose to develop the theory of fuzzy nabla dynamic equations on time scales. In this context, we introduce and study fuzzy nabla initial value problems on time scales. The rest of this paper is organized as follows. In Sect. 2, we present some definitions, properties, basic results relating to fuzzy sets, calculus of fuzzy functions, and time scales calculus. In Sect. 3, we introduce first type and second type nabla Hukuhara derivative of fuzzy functions on time scales. We establish the existenceuniqueness result for FNIVP with the help of contraction mapping principle and also obtain the existence result for FNIVP using Ascoli's and Schauder's fixed point theorems in Sect. 4.

\section{Preliminaries}

It is important to recall some basic results and definitions related to fuzzy calculus. Let $\Re_{k}\left(\Re^{n}\right)$ be the family of all convex compact nonempty subsets of $\Re^{n}$. Denote the set addition and scalar multiplication in $\Re_{k}\left(\Re^{n}\right)$ as usual. Then $\Re_{k}\left(\Re^{n}\right)$ satisfies the properties of a commutative semigroup [17] with addition of cancelation laws. Further, if $\alpha, \beta \in \Re$ and $S, T \in \Re_{k}\left(\Re^{n}\right)$, then

$$
\alpha(S+T)=\alpha S+\alpha T, \quad \alpha(\beta S)=(\alpha \beta) S, \quad 1 . S=S,
$$

and if $\beta, \alpha \geq 0$, then $(\beta+\alpha) S=\beta S+\alpha S$. Let $S$ and $T$ be two bounded nonempty subsets of $\Re^{n}$. By using the Pompeiu-Hausdorff metric, we define the distance between $S$ and $T$ as follows:

$$
d_{H}(S, T)=\max \left\{\sup _{s \in S} \inf _{t \in T}\|s-t\|, \operatorname{supinf}_{t \in T}\|s-t\|\right\} .
$$

Here, $\|\cdot\|$ is the Euclidean norm in $\Re^{n}$. Then $\left(\Re_{k}\left(\Re^{n}\right), d_{H}\right)$ becomes a separable and complete metric space [17]. 
Define

$$
\mathbb{E}_{n}=\left\{\mu: \Re^{n} \rightarrow[0,1] / \mu \text { satisfies }(\mathrm{a})-(\mathrm{d}) \text { below }\right\} \text {, where }
$$

(a) if $\exists$ a $t \in \Re^{n}$ such that $\mu(t)=1$, then $\mu$ is said to be normal,

(b) $\mu$ is fuzzy convex,

(c) $\mu$ is upper semicontinuous,

(d) the closure of $\left\{t \in \mathfrak{R}^{n} / \mu(t)>0\right\}=[\mu]^{0}$ is compact.

For $0<\lambda \leq 1$, denote $[\mu]^{\lambda}=\left\{t \in \Re^{n}: \mu(t) \geq \lambda\right\}$, then from the above conditions we have that the $\lambda$-level set $[\mu]^{\lambda} \in \Re_{k}\left(\Re^{n}\right)$.

We know that $[g(s, t)]^{\lambda}=g\left([s]^{\lambda},[t]^{\lambda}\right)$ for all $s, t \in \mathbb{E}_{n}$ and $g$ is continuous. The scalar multiplication $\odot$ and addition $\oplus$ of $s, t \in \mathbb{E}_{n}$ is defined as

$$
[s \oplus t]^{\lambda}=[s]^{\lambda}+[t]^{\lambda},[k \odot s]^{\lambda}=k[s]^{\lambda}, \quad \text { where } s, t \in \mathbb{E}_{n}, k \in \mathfrak{R}, 0 \leq \lambda \leq 1 \text {. }
$$

Define $D_{H}: \mathbb{E}_{n} \times \mathbb{E}_{n} \rightarrow[0, \infty)$ by

$$
D_{H}(s, t)=\sup _{0 \leq \lambda \leq 1} d_{H}\left([s]^{\lambda},[t]^{\lambda}\right)
$$

here $d_{H}$ is the Pompeiu-Hausdorff metric defined in $\Re_{k}\left(\Re^{n}\right)$. Then $\left(\mathbb{E}_{n}, D_{H}\right)$ is a complete metric space [17].

The following theorem extends the properties of addition and scalar multiplication of fuzzy number-valued functions $\left(\Re_{F}=\mathbb{E}_{1}\right)$ to $\mathbb{E}_{n}$.

\section{Theorem $1([3])$}

(a) If $\tilde{0}$ is the zero element in $\Re_{F}$, then $\hat{0}=(\tilde{0}, \tilde{0}, \ldots, \tilde{0})$ is the zero element in $\mathbb{E}_{n}$, i.e., $s \oplus \hat{0}=\hat{0} \oplus s=s \forall s \in \mathbb{E}_{n} ;$

(b) For any $s \in \mathbb{E}_{n}$ has no inverse with respect to ' $\oplus$ ';

(c) For any $\beta, \gamma \in \Re$ with $\beta, \gamma \geq 0$ or $\beta, \gamma \leq 0$ and $s \in \mathbb{E}_{n}$, then $(\beta+\gamma) \odot s=(\beta \odot s) \oplus(\gamma \odot s)$;

(d) For any $\beta \in \Re$ and $s, t \in \mathbb{E}_{n}$, we have $\beta \odot(s \oplus t)=(\beta \odot s) \oplus(\beta \odot t)$;

(e) For any $\beta, \gamma \in \mathfrak{R}$ and $s \in \mathbb{E}_{n}$, we have $\beta \odot(\gamma \odot s)=(\beta \gamma) \odot s$.

Let $S, T \in \mathbb{E}_{n}$. If $\exists$ a $R \in \mathbb{E}_{n}$ such that $S=T \oplus R$, then we say that $R$ is the $H$-difference of $S$ and $T$ and is denoted by $S \ominus_{h} T$. For any $S, T, R, U \in \mathbb{E}_{n}$ and $\alpha \in \Re$, the following hold:

(a) $D_{H}(S, T)=0 \Leftrightarrow S=T$;

(b) $D_{H}(\alpha S, \alpha T)=|\alpha| D_{H}(S, T)$;

(c) $D_{H}(S \oplus R, T \oplus R)=D_{H}(S, T)$;

(d) $D_{H}\left(S \ominus_{h} R, T \ominus_{h} R\right)=D_{H}(S, T)$;

(e) $D_{H}(S \oplus T, R \oplus U) \leq D_{H}(S, R)+D_{H}(T, U)$;

(f) $D_{H}\left(S \ominus_{h} T, R \ominus_{h} U\right) \leq D_{H}(S, R)+D_{H}(T, U)$

provided the $\mathrm{H}$-differences exist.

Now, we present some fundamental definitions and properties of Hukuhara derivative of fuzzy functions on the compact interval $I=[a, b], a, b \in \Re$. 
Remark 1 ([17]) A function $G$ is said to be differentiable if the multivalued mapping $G_{\lambda}$ is Hukuhara differentiable for all $\lambda \in[0,1]$ and

$$
\left[G_{\lambda}(\theta)\right]^{\prime}=\left[G^{\prime}(\theta)\right]^{\lambda}
$$

where $\left[G_{\lambda}\right]^{\prime}$ is the H-derivative of $G_{\lambda}$.

Definition 1 ([17]) A mapping $G$ is said to be strongly measurable if, for each $\lambda \in[0,1]$, the fuzzy function $G: I \rightarrow \Re_{k}\left(\Re^{n}\right)$ defined by $G_{\lambda}(\theta)=[G(\theta)]^{\lambda}$ is measurable.

Now, we present some fundamental definitions and results of time scales.

\section{Definition $2([10])$}

(a) Any nonempty closed subset of $\Re$ is defined as a time scale which is denoted by $\mathbb{T}$.

(b) $\rho: \mathbb{T} \rightarrow \mathbb{T}$ is the backward jump operator and $v: \mathbb{T} \rightarrow \mathbb{R}^{+}$, the graininess operator is defined by

$$
\rho(\theta)=\sup \left\{\theta_{0} \in \mathbb{T}: \theta_{0}<\theta\right\}, \quad v(\theta)=\theta-\rho(\theta) \quad \text { for } \theta \in \mathbb{T} .
$$

(c) If $\theta>\inf (\mathbb{T})$ and $\rho(\theta)=\theta$, then $\theta$ is called left dense otherwise left scattered.

(d) $\mathbb{T}_{k}=\mathbb{T}-\{m\}$, if $\mathbb{T}$ has a right scattered minimum $m$. Otherwise $\mathbb{T}_{k}=\mathbb{T}$.

(e) A mapping $g^{\rho}: \mathbb{T} \rightarrow \Re$ is defined by

$$
g^{\rho}(\theta)=g(\rho(\theta)) \quad \text { for each } \theta \in \mathbb{T} \text {, }
$$

where $g: \mathbb{T} \rightarrow \Re$ is a function.

(f) The interval in time scale $\mathbb{T}$ is defined by

$$
\mathbb{T}^{[a, b]}=\{\theta \in \mathbb{T}: a \leq \theta \leq b\}=[a, b] \cap \mathbb{T},
$$

and if ' $a$ ' is right dense, then

$$
\mathbb{T}_{k}^{[a, b]}=\mathbb{T}^{[a, b]},
$$

or if ' $a$ ' is right scattered, then

$$
\mathbb{T}_{k}^{[a, b]}=\mathbb{T}^{[\sigma(a), b]} .
$$

Definition 3 ([10]) Let $g: \mathbb{T} \rightarrow \Re$ be a function and $\theta \in \mathbb{T}_{k}$. Then $g^{\nabla}(\theta)$ exists as a number provided for any given $\epsilon>0, \exists$ a neighborhood $N_{\delta}$ of $\theta$ (i.e., $N_{\delta}=(\theta-\delta, \theta+\delta) \cap \mathbb{T}$ for some $\delta>0$ ) such that

$$
\left|\left[g(\rho(\theta))-g\left(\theta_{0}\right)\right]-g^{\nabla}(\theta)\left[\rho(\theta)-\theta_{0}\right]\right| \leq \epsilon\left|\rho(\theta)-\theta_{0}\right| \quad \text { for all } \theta_{0} \in N_{\delta} .
$$

Here, $g^{\nabla}(\theta)$ is called the nabla derivative of $g$ at $\theta$. Moreover, $g$ is said to be nabla (or Hilger) differentiable on $\mathbb{T}_{k}$, if $g^{\nabla}(\theta)$ exists $\forall \theta \in \mathbb{T}_{k}$. The function $g^{\nabla}: \mathbb{T}_{k} \rightarrow \Re$ is then called the nabla derivative of $g$ on $\mathbb{T}_{k}$. 
Definition 4 ([10]) A mapping $g: \mathbb{T} \rightarrow \Re$ is said to be regulated if its left sided limits exist and are finite at all ld-points (left dense points) in $\mathbb{T}$ and its right sided limits exist and are finite at all rd-points (right dense points) in $\mathbb{T}$.

Definition 5 ([10]) Let $g: \mathbb{T} \rightarrow \Re$ be a function. $g$ is said to be ld-continuous if it is continuous at each ld-point in $\mathbb{T}$ and $\lim _{\theta_{0} \rightarrow \theta^{+}} g(\theta)$ exists as a finite number for all rd-points in $\mathbb{T}$.

Theorem 2 (Ascoli's theorem on $\mathbb{T}[34])$ Assume $Y \subseteq C_{l d f}\left(\mathbb{T}^{[a, b]}, \Re\right)$. Then $Y$ is bounded and equi-continuous iff $Y$ is relatively compact.

Theorem 3 (Schauder's fixed point theorem [22]) Let $V$ be a normed linear space and $X$ be a bounded, closed, and convex subset of $V$. If $G: V \rightarrow V$ is compact, then $G$ has at least one fixed point.

\section{Nabla Hukuhara differentiability and integrability}

In this section, we present first and second type nabla Hukuhara derivatives, fuzzy nabla integral of fuzzy functions on time scales, and their properties.

Definition 6 ([19]) Suppose that $G: \mathbb{T}^{[a, b]} \rightarrow \mathbb{E}_{n}$ is a fuzzy function and $\theta \in \mathbb{T}_{k}^{[a, b]}$. Let $G^{\nabla^{h}}(\theta)$ be an element of $\mathbb{E}_{n}$. For any given $\epsilon>0, \exists$ a neighborhood $N_{\mathbb{T}}[a, b]$ of $\theta$, and for some $\delta>0$ such that

$$
\begin{aligned}
& D_{H}\left[\left(G(\theta+\hbar) \ominus_{h} G(\rho(\theta)),(\hbar+v(\theta)) \odot G^{\nabla^{h}}(\theta)\right] \leq \epsilon|\hbar+v(\theta)|,\right. \\
& D_{H}\left[\left(G(\rho(\theta)) \ominus_{h} G(\theta-\hbar),(\hbar-v(\theta)) \odot G^{\nabla^{h}}(\theta)\right] \leq \epsilon|\hbar-v(\theta)|,\right.
\end{aligned}
$$

for all $\theta-\hbar, \theta+\hbar \in N_{\mathbb{T}[a, b]}$ with $0<h<\delta$, where $\nu(\theta)=\theta-\rho(\theta)$. Then $G$ is called nabla Hukuhara form-I (nabla-h) differentiable at $\theta$ and is denoted by $G^{\nabla^{h}}(\theta)$.

The above definition does not exist if the fuzzy function has a decreasing diameter. So, in order to overcome this circumstance, we introduce and study the second type nabla Hukuhara derivative for fuzzy functions on time scales where the results exist for the functions which have a decreasing diameter.

Definition 7 ([19]) Suppose that $G: \mathbb{T}^{[a, b]} \rightarrow \mathbb{E}_{n}$ is a fuzzy function and $\theta \in \mathbb{T}_{k}^{[a, b]}$. Let $G^{\nabla^{s h}}(\theta)$ be an element of $\mathbb{E}_{n}$ for any given $\epsilon>0, \exists$ a neighborhood $N_{\mathbb{T}^{[a, b]}}$ of $\theta$, and for some $\delta>0$ such that

$$
\begin{aligned}
& D_{H}\left[(G(\rho(\theta)) \ominus G(\theta+\hbar)),-(\hbar+v(\theta)) \odot G^{\nabla^{s h}}(\theta)\right] \leq \epsilon|-(\hbar+v(\theta))|, \\
& D_{H}\left[\left(G(\theta-\hbar) \ominus_{h} G(\rho(\theta)),-(\hbar-v(\theta)) \odot G^{\nabla^{s h}}(\theta)\right] \leq \epsilon|-(\hbar-v(\theta))|,\right.
\end{aligned}
$$

for all $\theta-\hbar, \theta+\hbar \in N_{\mathbb{T}^{[}[a, b]}$ with $0<h<\delta$, where $v(\theta)=\theta-\rho(\theta)$. Then $G$ is called nabla Hukuhara form-II differentiable or nabla-sh (symbolically $\nabla^{\text {sh }}$-differentiable) at $\theta$ and is denoted by $G^{\nabla^{\text {sh }}}(\theta)$. We consider only right limit at left scattered points and one-sided limit at the end points of $\mathbb{T}_{k}^{[a, b]}$. 
Lemma 1 ([19]) Let $G: \mathbb{T}^{[a, b]} \rightarrow \mathbb{E}_{n}$ be a fuzzy function. If $G: \mathbb{T}^{[a, b]} \rightarrow \mathbb{E}_{n}$ is continuous at $\theta$ and $\theta$ is left scattered, then

(a) $G: \mathbb{T}^{[a, b]} \rightarrow \mathbb{E}_{n}$ is nabla-h differentiable at $\theta \in \mathbb{T}_{k}^{[a, b]}$ with

$$
G^{\nabla^{h}}(\theta)=\frac{1}{v(\theta)} \odot\left(G(\theta) \ominus_{h} G(\rho(\theta))\right)
$$

provided $G(\theta) \ominus_{h} G(\rho(\theta))$ exists.

or

(b) $G: \mathbb{T}^{[a, b]} \rightarrow \mathbb{E}_{n}$ is nabla-sh differentiable at $\theta \in \mathbb{T}_{k}^{[a, b]}$ with

$$
G^{\nabla^{s h}}(\theta)=\frac{-1}{v(\theta)} \odot\left(G(\rho(\theta)) \ominus_{h} G(\theta)\right),
$$

provided $G(\rho(\theta)) \ominus_{h} G(\theta)$ exists.

or

(c) $G: \mathbb{T}^{[a, b]} \rightarrow \mathbb{E}_{n}$ is nabla differentiable at $\theta \in \mathbb{T}_{k}^{[a, b]}$ with

$$
G^{\nabla}(\theta)=\frac{1}{v(\theta)} \odot\left(G(\theta) \ominus_{h} G(\rho(\theta))\right)=\frac{-1}{v(\theta)} \odot\left(G(\rho(\theta)) \ominus_{h} G(\theta)\right) \in \Re^{n},
$$

provided $G(\rho(\theta)) \ominus_{h} G(\theta)$ and $G(\theta) \ominus_{h} G(\rho(\theta))$ both exist.

Theorem 4 ([19]) If G, $H: \mathbb{T}^{[a, b]} \rightarrow E^{n}$ are nabla integrable, then the following hold:

(a) $\int_{a}^{b}[G(\tau) \oplus H(\tau)] \nabla \tau=\int_{a}^{b} G(\tau) \nabla \tau \oplus \int_{a}^{b} H(\tau) \nabla \tau$.

(b) $\int_{a}^{b} \alpha G(\tau) \nabla \tau=\alpha \int_{a}^{b} G(\tau) \nabla \tau, \alpha \in \Re$.

(c) $\int_{a}^{b} G(\tau) \nabla \tau=\int_{a}^{c} G(\tau) \nabla \tau \oplus \int_{c}^{b} G(\tau) \nabla \tau$.

(d) $\int_{a}^{a} G(\tau) \nabla \tau=\hat{0}$.

(e) If $g \in S_{G}\left(\mathbb{T}^{[a, b]}\right)$, then $D_{H}(G(\cdot), \hat{0}): \mathbb{T}^{[a, b]} \rightarrow R^{+}$is nabla integrable and

$$
D_{H}\left(\int_{a}^{b} G(\tau) \nabla \tau, \hat{0}\right) \leq \int_{a}^{b} D_{H}(G(\tau), \hat{0}) \nabla \tau .
$$

(f) If $g \in S_{G}\left(\mathbb{T}^{[a, b]}\right)$ and $h \in S_{H}\left(\mathbb{T}^{[a, b]}\right)$ imply that $g, h \in C_{l d f}\left(\mathbb{T}^{[a, b]}\right)$ respectively, then $D_{H}(G(\cdot), H(\cdot)): \mathbb{T}^{[a, b]} \rightarrow \Re^{+}$is nabla integrable and

$$
D_{H}\left(\int_{a}^{b} G(\tau) \nabla \tau, \int_{a}^{b} H(\tau) \nabla \tau\right) \leq \int_{a}^{b} D_{H}(G(\tau), H(\tau)) \nabla \tau,
$$

where the set of all fuzzy ld-continuous functions is defined by

$$
C_{l d f}=C_{l d f}\left(\mathbb{T}^{[a, b]}\right)=C_{l d f}\left(\mathbb{T}^{[a, b]}, \mathbb{E}_{n}\right) .
$$

Theorem 5 ([19]) Suppose that $G: \mathbb{T}^{[a, b]} \rightarrow \mathbb{E}_{n}$ is ld-continuous and if $\theta_{0} \in \mathbb{T}^{[a, b]}$, then

(a) $\mathcal{G}$ defined by

$$
\mathcal{G}(\theta)=X_{0} \oplus \int_{\theta_{0}}^{\theta} G(\tau) \nabla \tau \quad \text { for } \theta \in \mathbb{T}^{[a, b]} \text { and } X_{0} \in \mathbb{E}_{n}
$$

is nabla-h differentiable and $\mathcal{G}^{\nabla^{h}}(\theta)=G(\theta)$ a.e. on $\mathbb{T}^{[a, b]}$. 
(b) $\mathcal{G}$ defined by

$$
\mathcal{G}(\theta)=X_{0} \ominus_{h}(-1) \int_{\theta_{0}}^{\theta} G(\tau) \nabla \tau \quad \text { for } \theta \in \mathbb{T}^{[a, b]} \text { and } X_{0} \in \mathbb{E}_{n}
$$

is nabla-sh differentiable and $\mathcal{G}^{\nabla^{\text {sh }}}(\theta)=G(\theta)$ a.e. on $\mathbb{T}^{[a, b]}$.

\section{Main results}

In this section, we introduce a fuzzy nabla initial value problem on time scales and obtain the solution under two types of Hukuhara differentiability. Let us consider the fuzzy nabla initial value problems $(F N I V P)$ on time scales as

$$
\begin{array}{ll}
u^{\nabla h}(\theta)=G(\theta, u), & u\left(\theta_{0}\right)=u_{0}, \quad \theta_{0} \geq 0, \\
u^{\nabla^{s h}}(\theta)=G(\theta, u), & u\left(\theta_{0}\right)=u_{0}, \quad \theta_{0} \geq 0,
\end{array}
$$

where $G \in C_{l d f}\left(I_{\mathbb{T}}, \mathbb{E}_{n}\right), I_{\mathbb{T}}=\mathbb{T}^{\left[\theta_{0}, \theta_{0}+a\right]}, a>0$.

Lemma 2 A mapping $u: I_{\mathbb{T}} \rightarrow \mathbb{E}_{n}$ is a solution of FNIVP (5) iff it is ld-continuous and satisfies the fuzzy nabla integral equation

$$
u^{\nabla^{h}}(\theta)=u_{0} \oplus \int_{\theta_{0}}^{\theta} G(\tau, u(\tau)) \nabla \tau, \quad \theta \in I_{\mathbb{T}}
$$

and $u$ is a solution of FNIVP (5) iff it is ld-continuous and satisfies the fuzzy nabla integral equation

$$
u^{\nabla^{s h}}(\theta)=u_{0} \oplus(-1) \int_{\theta_{0}}^{\theta} G(\tau, u(\tau)) \nabla \tau, \quad \theta \in I_{\mathbb{T}} .
$$

Proof Suppose that $u(\theta)$ is a solution of FNIVP (5). Then

$$
u^{\nabla^{h}}(\theta)=G(\theta, u(\theta)), \quad u\left(\theta_{0}\right)=u_{0} .
$$

Taking fuzzy nabla integral on (5)

$$
\begin{gathered}
\int_{\theta_{0}}^{\theta} u^{\nabla^{h}}(\tau) \nabla \theta=\int_{\theta_{0}}^{\theta} G(\tau, u(\tau)) \nabla \tau \\
\Longrightarrow u(\theta) \ominus_{h} u\left(\theta_{0}\right)=\int_{\theta_{0}}^{\theta} G(\tau, u(\tau)) \nabla \tau \\
\Longrightarrow \quad u(\theta)=u_{0} \oplus \int_{\theta_{0}}^{\theta} G(\tau, u(\tau)) \nabla \tau .
\end{gathered}
$$

Conversely, suppose that $u$ satisfies the fuzzy integral equation (7). Clearly, $u\left(\theta_{0}\right)=u_{0}$, and from Theorem ( 5)(a), we have $u^{\nabla^{h}}(\theta)=G(\theta, u(\theta))$. Similarly, we prove the other part.

Now, with the help of Banach contraction mapping principle, we will prove that if $G(\theta, u)$ satisfies the Lipschitz condition, then FNIVPs (5) and (6) have a unique solution on $I_{\mathbb{T}}$. 
Theorem 6 Let $G: I_{\mathbb{T}} \times \mathbb{E}_{n} \rightarrow \mathbb{E}_{n}$ be ld-continuous and suppose that there exists $M>0$ such that

$$
D_{H}(G(\theta, \psi), G(\theta, \phi)) \leq M D_{H}(\psi, \phi), \quad \forall \theta \in I_{\mathbb{T}}, u, v \in \mathbb{E}_{n},
$$

then FNIVPs (5) and (6) have a unique solution on $I_{\mathbb{T}}$.

Proof Let $C_{l d f}\left(I_{\mathbb{T}}, \mathbb{E}_{n}\right)$ be the set of all ld-continuous functions from $I_{\mathbb{T}}$ to $\mathbb{E}_{n}$, where $I_{\mathbb{T}} \in$ $\mathbb{T}^{[a, b]}$. Define the metric

$$
\chi(\psi, \phi)=\sup _{\theta \in I_{\mathbb{T}}} D_{H}(\psi(\theta), \phi(\theta))
$$

for all $\psi, \phi \in C_{l d f}\left(I_{\mathbb{T}}, \mathbb{E}_{n}\right)$. Since $\left(D_{H}, \mathbb{E}_{n}\right)$ is a complete metric space, it is evident that $\left(C_{\text {ldf }}\left(I_{\mathbb{T}}, \mathbb{E}_{n}\right), \chi\right)$ is also a complete metric. For any $\psi \in C_{\text {ldf }}\left(I_{\mathbb{T}}, \mathbb{E}_{n}\right)$, define the operator $F$ on $I_{\mathbb{T}}$ as

$$
F[\psi(\theta)]=u_{0} \oplus \int_{\theta_{0}}^{\theta} G(\tau, \psi(\tau)) \nabla \tau, \quad \forall \theta \in I_{\mathbb{T}}
$$

Now, we show that $F \psi \in C_{l d f}\left(I_{\mathbb{T}}, \mathbb{E}_{n}\right)$ employing ld-continuity and nabla integral properties. Let $\theta, \theta_{0} \in I_{\mathbb{T}}$

$$
\begin{aligned}
D_{H}\left(F \psi(\theta), F \psi\left(\theta_{0}\right)\right) & =D_{H}\left(u_{0} \oplus \int_{\theta_{0}}^{\theta} G(\tau, \psi(\tau)) \nabla \tau, u_{0} \oplus \int_{\theta_{0}}^{\theta_{0}} G(\tau, \psi(\tau)) \nabla \tau\right) \\
& =D_{H}\left(\int_{\theta_{0}}^{\theta} G(\tau, \psi(\tau)) \nabla \tau, \int_{\theta_{0}}^{\theta_{0}} G(\tau, \psi(\tau)) \nabla \tau\right) \\
& \leq \int_{\theta_{0}}^{\theta} D_{H}(G(\tau, \psi(\tau)) \nabla \tau, \hat{0}) \\
& \leq \int_{\theta_{0}}^{\theta}\|G(\tau, \psi(\tau))\| \nabla \tau .
\end{aligned}
$$

Since $G$ satisfies the Lipschitz condition, $G$ is bounded and

$$
\int_{\theta_{0}}^{\theta}\|G(\tau, \psi(\tau))\| \nabla \tau \leq M\left|\theta-\theta_{0}\right|
$$

Hence, $D_{H}\left(F \psi(\theta), F \psi\left(\theta_{0}\right)\right) \leq M\left|\theta-\theta_{0}\right|$. Therefore, $F \psi \in C_{l d f}\left(I_{\mathbb{T}}, \mathbb{E}_{n}\right)$. Now, consider

$$
\begin{aligned}
\chi(F \psi, F \phi) & =\sup _{I_{\mathbb{T}}} D_{H}\left(\int_{\theta_{0}}^{\theta} G(\tau, \psi(\tau)) \nabla \tau, \int_{\theta_{0}}^{\theta} G(\tau, \phi(\tau)) \nabla \tau\right) \\
& \leq \int_{\theta_{0}}^{\theta_{0}+a} D_{H}(G(\tau, \psi(\tau)), G(\tau, \phi(\tau)) \nabla \tau \\
& \left.\leq \int_{\theta_{0}}^{\theta_{0}+a} M D_{H}(\psi(\tau)), \phi(\tau)\right) \nabla \tau \\
& \leq a M \chi(\psi, \phi) .
\end{aligned}
$$


Choose $I_{\mathbb{T}}$ such that $a M<1$. Therefore, $F$ is a contraction mapping. Thus, from the Banach contraction mapping principle there exists a unique fixed point of $F$, say $\tilde{u}$, and this fixed point is the unique solution of FNIVP (5) on $I_{\mathbb{T}}$. Similarly, we can prove the existence and uniqueness solution for FNIVP (6) on $I_{\mathbb{T}}$.

In the following theorem, we establish the existence of solutions for FNIVPs (5) and (6) using Ascoli's theorem and Schauder's fixed point theorem.

Theorem 7 Suppose that $G \in C_{\text {ldf }}\left(I_{\mathbb{T}} \times \mathbb{E}_{n}, \mathbb{E}_{n}\right)$ and $D_{H}(G(\theta, u), \hat{0}) \leq K, \theta \in I_{\mathbb{T}}, u \in \mathbb{E}_{n}$. Then FNIVPs (5) and (6) have at least one solution on $I_{\mathbb{T}}$.

Proof First, we consider FNIVP (5) and we prove that it has at least one solution. Let $\mathcal{B}$ be a bounded set in $C_{l d f}\left(I_{\mathbb{T}}, \mathbb{E}_{n}\right)$. The set $F \mathcal{B}=\{[F u]: u \in \mathcal{B}\}$ is totally bounded iff it is equicontinuous, and for $\theta \in I_{\mathbb{T}}$, the set

$$
[F \mathcal{B}](\theta)=\left\{[F u](\theta): \theta \in I_{\mathbb{T}}\right\}
$$

is a totally bounded subset of $\mathbb{E}_{n}$, where $F u(\theta)=u_{0} \oplus \int_{\theta_{0}}^{\theta} G(\tau, u(\tau)) \nabla \tau$. For any $\theta_{1}, \theta_{2} \in I_{\mathbb{T}}$, $\theta_{1} \leq \theta_{2}$ and $u \in \mathcal{B}$, we have

$$
\begin{aligned}
D_{H}\left(F u\left(\theta_{1}\right), F u\left(\theta_{2}\right)\right) & =D_{H}\left(u_{0} \oplus \int_{\theta_{0}}^{\theta_{1}} G(\tau, u(\tau)) \nabla \tau, u_{0} \oplus \int_{\theta_{0}}^{\theta_{2}} G(\tau, u(\tau)) \nabla \tau\right) \\
& =D_{H}\left(\int_{\theta_{0}}^{\theta_{1}} G(\tau, u(\tau)) \nabla \tau, \int_{\theta_{0}}^{\theta_{2}} G(\tau, u(\tau)) \nabla \tau\right) \\
& \leq \int_{\theta_{1}}^{\theta_{2}} D_{H}(G(\tau, u(\tau)), \hat{0}) \nabla \tau \\
& \leq\left|\theta_{2}-\theta_{1}\right| \max _{\theta \in I_{\mathbb{T}}} D_{H}(G(\tau, u(\tau)), \hat{0}) \\
& \leq\left|\theta_{2}-\theta_{1}\right| K .
\end{aligned}
$$

Therefore, $F u$ is equicontinuous on $I_{\mathbb{T}}$. For fixed $\theta \in I_{\mathbb{T}}$, we have

$$
D_{H}\left(F u(\theta), F u\left(\theta_{1}\right)\right) \leq\left|\theta-\theta_{1}\right| K, \quad \forall \theta_{1} \in I_{\mathbb{T}}, u \in \mathcal{B} .
$$

Hence, the set $\{[[F u](\theta): u \in \mathcal{B}]\}$ is totally bounded in $\mathbb{E}_{n}$. From Theorem 2 , we have $F \mathcal{B}$ is a relatively compact subset of $C_{l d f}\left(I_{\mathbb{T}}, \mathbb{E}_{n}\right)$. Define the ball $\mathcal{B}=\left\{u \in C_{\text {ldf }}\left(I_{\mathbb{T}}, \mathbb{E}_{n}\right) \ni \chi(u, \hat{0}) \leq\right.$ $a K\}$ in the metric space $\left(C_{l d f}\left(I_{\mathbb{T}}, \mathbb{E}_{n}\right), \chi\right)$. For $u \in C_{l d f}\left(I_{\mathbb{T}}, \mathbb{E}_{n}\right)$, consider

$$
\begin{aligned}
D_{H}\left(F u(\theta), F u\left(\theta_{0}\right)\right) & =D_{H}\left(u_{0} \oplus \int_{\theta_{0}}^{\theta} G(\tau, u(\tau)) \nabla \tau, u_{0}\right) \\
& =\int_{\theta_{0}}^{\theta} D_{H}(G(\tau, u(\tau)) \nabla \tau, \hat{0}) \\
& \leq \int_{\theta_{0}}^{\theta} K \nabla \tau \\
& \leq\left|\theta-\theta_{0}\right| K \leq a K .
\end{aligned}
$$


Defining $\tilde{0}: I_{\mathbb{T}} \rightarrow \mathbb{E}_{n}$ such that $\tilde{0}(\theta)=\hat{0}, \theta \in I_{\mathbb{T}}$, we get

$$
\chi(F u, F \hat{0})=\sup _{\theta \in I_{\mathbb{T}}} D_{H}((F u)(\theta),(F \tilde{0})(\theta)) \leq a K .
$$

Therefore, $F \mathcal{B}$ is contained in $\mathcal{B}$. Since $F$ is compact and then from Theorem (3), $F$ has a fixed point and this fixed point is a solution of (5). Hence, FNIVP (5) has at least one solution on $\theta \in I_{\mathbb{T}}$. In a similar way, we can prove the existence of solution for FNIVP (6) by defining

$$
F u(\theta)=u_{0} \oplus(-1) \int_{\theta_{0}}^{\theta} G(\tau, u(\tau)) \nabla \tau .
$$

\section{Conclusions}

In this paper, first we introduced fuzzy nabla initial value problems (FNIVP) on time scales under Hukuhara differentiability and represented their solutions as integral equations. Further, we established the existence and uniqueness of solution for FNIVP on time scales using the Banach contraction mapping principle and also obtained the existence result using Ascoli's and Schauder's fixed point theorems.

Funding

No funding

Availability of data and materials

Data sharing not applicable to this article as no data sets were generated or analyzed during the current study.

\section{Competing interests}

The authors declare that they have no competing interest.

\section{Authors' contributions}

All authors contributed equally and significantly in writing this article. All authors read and approved the final manuscript.

\section{Author details}

${ }^{1}$ Department of Mathematics, Koneru Lakshmaiah Education Foundation, KL Deemed to be University, Vaddeswaram, India. ${ }^{2}$ Department of Mathematics, Acharya Nagarjuna University, Guntur, India. ${ }^{3}$ Department of Mathematics, College of Natural and Computational Sciences, Wollega University, Nekemte, Ethiopia.

\section{Publisher's Note}

Springer Nature remains neutral with regard to jurisdictional claims in published maps and institutional affiliations.

Received: 9 November 2018 Accepted: 14 June 2019 Published online: 04 July 2019

References

1. Agarwal, R.P., Bohner, M.: Basic calculus on time scales and some of its applications. Results Math. 35, 3-22 (1999)

2. Agarwal, R.P., Bohner, M., Li, T.: Oscillatory behavior of second-order half-linear damped dynamic equations. Appl. Math. Comput. 254, 408-418 (2015)

3. Anastassiou, G.A., Gal, S.G.: On a fuzzy trigonometric approximation theorem of Weierstrass-type. J. Fuzzy Math. 9(3), 701-708 (2001)

4. Anderson, D.R., Tisdell, C.C.: Alternative solutions of inhomogeneous second-order linear dynamic equations on time scales. J. Differ. Equ. Appl. 17(10), 1487-1498 (2011)

5. Atici, F.M., Daniel Biles, C., Lebedinsky, A.: An application of time scales to economics. Math. Comput. Model. 43 $718-726$ (2006)

6. Atici, F.M., Uysal, F.: A production-inventory model of HMMS model on time scales. Appl. Math. Lett. 21(3), 236-243 (2008)

7. Bede, B., Gal, S.G.: Generalizations of the differentiability of fuzzy-number-valued functions with applications to fuzzy differential equations. Fuzzy Sets Syst. 151, 581-599 (2005)

8. Bohner, M., Hassan, T.S., Li, T.: Fite-Hille-Wintner-type oscillation criteria for second-order half-linear dynamic equations with deviating arguments. Indag. Math. 29, 548-560 (2018)

9. Bohner, M., Li, T.: Kamenev-type criteria for nonlinear damped dynamic equations. Sci. China Math. 58(7), 1445-1452 (2015) 
10. Bohner, M., Peterson, A.: Dynamic Equations on Time Scales: An Introduction with Applications. Springer, Birkhauser (2001)

11. Eloe, P.W., Henderson, J.: Boundary value problems for dynamic equations on time scales. In: Nonlinear Interpolation and Boundary Value Problems, pp. 181-224 (2016)

12. Gao, J., Wang, Q.R., Zhang, L.W.: Existence and stability of almost-periodic solutions for cellular neural networks with time-varying delays in leakage terms on time scales. Appl. Math. Comput. 237, 639-649 (2015)

13. Hilger, S.: Analysis on measure chains - a unified approach to continuous and discrete calculus. Results Math. 18, 18-56 (1990)

14. Hong, S.: Differentiability of multivalued functions on time scales and applications to multivalued dynamic equations. Nonlinear Anal. 71, 3622-3637 (2009)

15. Hukuhara, M.: Intégration des applications mesurables dont la valeur est un compact convexe. Funkc. Ekvacioj 10 205-223 (1967)

16. Jackson, B.J.: Adaptive control in the nabla setting. Neural Parallel Sci. Comput. 16, 253-272 (2008)

17. Kaleva, O.: Fuzzy differential equations. Fuzzy Sets Syst. 24, 301-317 (1987)

18. Leelavathi, R., Suresh Kumar, G., Murty, M.S.N.: Nabla integral for fuzzy functions on time scales. Int. J. Appl. Math. 31(5), 669-678 (2018)

19. Leelavathi, R., Suresh Kumar, G., Murty, M.S.N.: Nabla Hukuhara differentiability for fuzzy functions on time scales. IAENG Int. J. Appl. Math. 49(1), 114-121 (2019)

20. Li, T., Saker, S.H.: A note on oscillation criteria for second-order neutral dynamic equations on isolated time scales. Communications in Nonlinear Science and Numerical Simulation. 19, 4185-4188 (2014)

21. Liu, B., Do, Y., Batarfi, H.A., Alsaadi, F.E.: Almost periodic solution for a neutral-type neural networks with distributed leakage delays on time scales. Neurocomputing 173, 921-929 (2016)

22. Lloyd, N.G.: Degree Theory. Cambridge Tracts in Mathematics. Cambridge University Press, Cambridge (1978)

23. Lupulescu, $\vee$.: Hukuhara differentiability of interval-valued functions and interval differential equations on time scales. Inf. Sci. 248, 50-67 (2013)

24. Malinowski, M.T.: Interval differential equations with a second type Hukuhara derivative. Appl. Math. Lett. 24 2118-2123 (2011)

25. Malinowski, M.T.: Interval Cauchy problem with a second type Hukuhara derivative. Inf. Sci. 213, 94-105 (2012)

26. Puri, M.L., Ralescu, D.A.: Differentials for fuzzy functions. J. Math. Anal. Appl. 91, 552-558 (1983)

27. Stefanini, L., Bede, B.: Generalized Hukuhara differentiability of interval-valued functions and interval differential equations. Nonlinear Anal., Theory Methods Appl. 71, 1311-1328 (2009)

28. Vasavi, C.H., Suresh Kumar, G., Murty, M.S.N.: Fuzzy Hukuhara delta differential and applications to fuzzy dynamic equations on time scales. J. Uncertain Syst. 10(1), 163-180 (2016)

29. Vasavi, C.H., Suresh Kumar, G., Murty, M.S.N.: Fuzzy dynamic equations on time scales under second type Hukuhara delta derivative. Int. J. Chem. Sci. 14(1), 49-66 (2016)

30. Vasavi, C.H., Suresh Kumar, G., Murty, M.S.N.: Generalized differentiability and integrability for fuzzy set-valued functions on time scales. Soft Comput. 20, 1093-1104 (2016)

31. Vasavi, C.H., Suresh Kumar, G., Murty, M.S.N.: Fuzzy dynamic equations on time scales under generalized delta derivative via contractive-like mapping principles. Indian J. Sci. Technol. 9(25), 1-6 (2016)

32. Zadeh, L.A.: Fuzzy sets. Inf. Control 8, 338-353 (1965)

33. Zafer, A.: On oscillation and nonoscillation of second-order dynamic equations. Appl. Math. Lett. 22(1), 136-141 (2009)

34. Zaidi, A.H.: Existence of solutions and convergence results for dynamic initial value problems using lower and upper solutions. Electron. J. Differ. Equ. 161, 1 (2009)

35. Zhang, C., Agarwal, R.P., Bohner, M., Li, T.: Oscillation of fourth-order delay dynamic equations. Sci. China Math. 58, 143-160 (2015)

36. Zhang, S., Sun, J.: Stability of fuzzy differential equations with the second type of Hukuhara derivative. IEEE Trans. Fuzzy Syst. 23(4), 1323-1328 (2015)

\section{Submit your manuscript to a SpringerOpen ${ }^{\circ}$ journal and benefit from:}

- Convenient online submission

- Rigorous peer review

- Open access: articles freely available online

- High visibility within the field

- Retaining the copyright to your article

Submit your next manuscript at $\boldsymbol{s p r i n g e r o p e n . c o m ~}$ 